\title{
A Graphics Platform for the Realization of the Algorithms of Automatic Drawing and the Computer Photographic Straightening
}

\author{
P.PALKA \& E.CAVALLINI \\ G. d'Annunzio University, Pescara, Italy
}

ABSTRACT: The research carried out by Computer Science Laboratory Labinf (INGEO Department-G. d' Annunzio University), coordinated by the professor Palka led to the study and realisation of a graphics platform for automatic drawing in programming language Pascal. The software Fotorad can be used for architectural survey and it's based on the homographic transformation of projective and analytical geometry. KEYWORD: Automatic drawing; graphic platform; computer photographic straightening; software Fotorad

\section{STRAIGHTENING THE PHOTOGRAPHIC COMPUTER}

\subsection{The current status}

In the last years, the Italian peninsula has been characterized by a number of seismic events of large magnitude and its historical, architectural and artistic buildings have been destroyed and damaged.

These events have leaded to consider the issue, especially the fragility of minor historical centers and towns that are important to local economic and cultural resources and to preserve and enhance them for the present and future generations.

These reasons have brought to the awareness and to the need to conservation action and recovery through a methodology that is able to represent the morphological and structural characteristics of damaged buildings and constructions The considerable wealth leads to intervene very often with low cost and qualitative results in the relief operations and Return: The traditional tools such as 3D laser scanner and photogrammetry have high intervention costs and are often inadequate with respect to the shape of the historical centers that are often perched on the heights with scenes building overlooking the narrow and irregular streets.

For this reason it is often used photogrammetry [1] to a single frame, also called straightening photo. It is well known, in fact, that by taking an image, it is possible in various ways to obtain opportunely metric data using the program to perform the socalled straightening photo for photoplan that allows an orthogonal vision of a photo taken anyway.

From this photo, you can get a refund by importing it into a vector drawing software Auto $\mathrm{CAD}$, and underline the architectural highlights. It remains clear that in doing so, you lose the perspective because any projections on the facade as cornices, frames and balconies are strongly deformed instead of a photo which is taken in perspective.

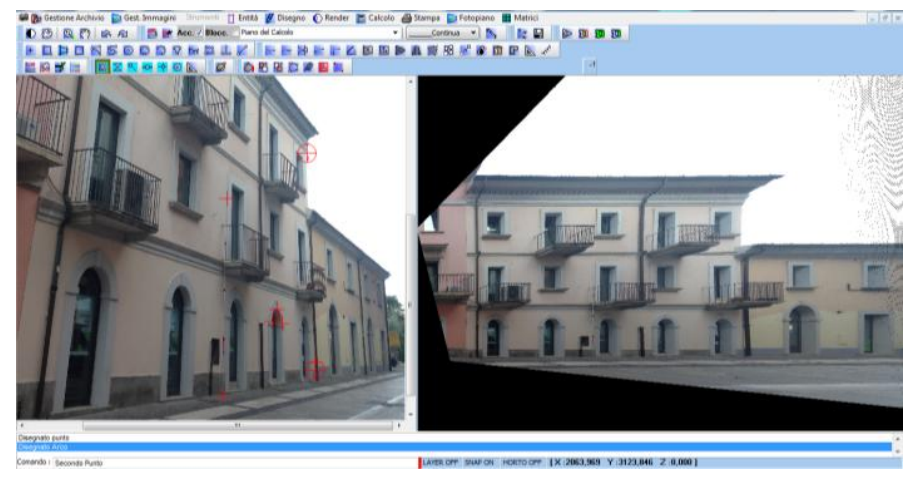

Figure 1. Straightening photo of a facade in the historical center of Pescara.

\section{THE FIELD OF RESEARCH}

\subsection{The software Fotorad}

From these requirements, at the Laboratory of Computer Science (department INGEO-University "G. d' Annunzio" of Chieti-Pescara), we committed ourselves in delivering a software called Fotorad, in a Windows environment, that would combine the work done separately from different software in one software that would allow even the inverse control of a refund.

The software presents the algorithms of automatic drawing in Pascal designed and manufactured without the use of third-party libraries, therefore, be easily customized to support the importance of the architecture. 
All this has allowed us to experience the goodness of the algorithms used in refining writing to get a more accurate calculation and simplification of procedures in the field in time realizing a program of rectification computerized image of the integrated platform proprietary graphic of automatic drawing.

It is known that one of the most important aspects related to the subjects of architecture, and it is a science of representation, which, making use of classic design rules-based projective known and well-established over time, allows you to draw its various elements, which form the profile.

Tracing the history of the various human achievements, together with the numerous anthropological phenomena, also studying architecture have undergone evolutionary moments that involved, so remarkable and rapid theorized concepts from the distant past and its forms of expression.

Referring to our times, the new way of representing the architecture uses a different kind of support, which is connected to the introduction of computer graphics using software linked to the design called digital.

However, in the full knowledge and understanding of the evolutionary path of how to represent a pattern, and not reject the results, the question arises whether the same rules projective used for traditional drawing on paper, worth also for the new type of digital design and if, in the case of a positive answer, the same can be applied in the same manner.

The answer is certainly not very easy because it is involved in numerous and varied concepts, including those relating to the new semantics.

No doubt the same trend has affected the language, with the introduction of new terms, sometimes not unequivocal, that are often different from basic theory.

With the new and different conditions of representation it is important not only the use of modern instruments, based, as noted, on icons that intuitively lead the operator to perform almost unconsciously the command that it represents, but, beyond the limits of read-only icon, he must also have knowledge of basic scientific concepts that are implied in the iconographic representation.

And this is the reason that led us to the creation of software for the detection of architectural facades that are most important for the purposes of preservation, renovation and restoration, and that presents the greatest difficulty of measuring regarding heights.

The software is based on the realization of an algorithm based on the homographic transformation [4] between floors in which it is necessary to identify eight transformation parameters to build a matrix with which to locate all points of the facade to be detected. The identification of the eight parameters can be done using two methods: the first based on the support points and the second based on the lines of escape.

The correct return of the various architectural elements of a façade was decided to split the window video in two windows: the first is contained entirely in any case the image photographed architecture under investigation, and from the other, the return vector in orthogonal projection of the same elements. It is just this last mode of operation that gives the software a peculiarity.

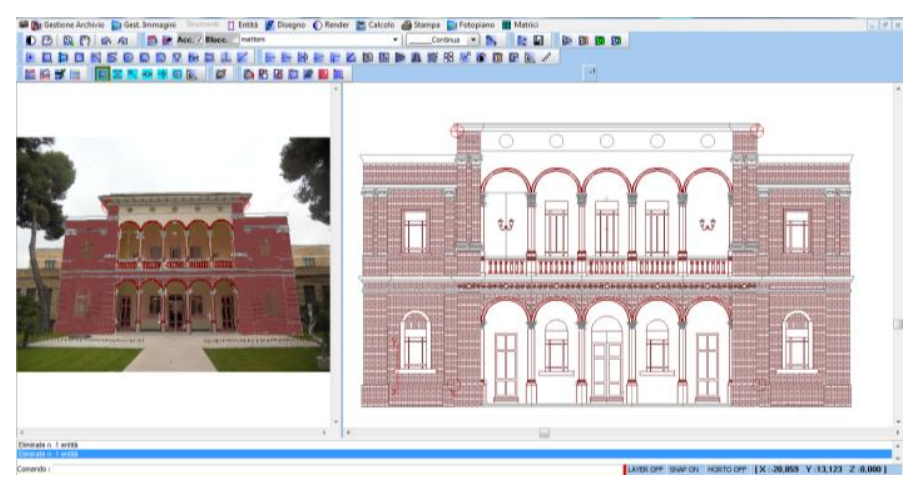

Figure 2. The double window to return the software Fotorad.

The second method, the lines of flight, then, can be done in two ways: in the first case, through the classical geometry of Gaspard Monge, [2] that identifies the two vanishing points towards which the parallel lines appear to converge the facade and in the second, however, through analytic geometry.

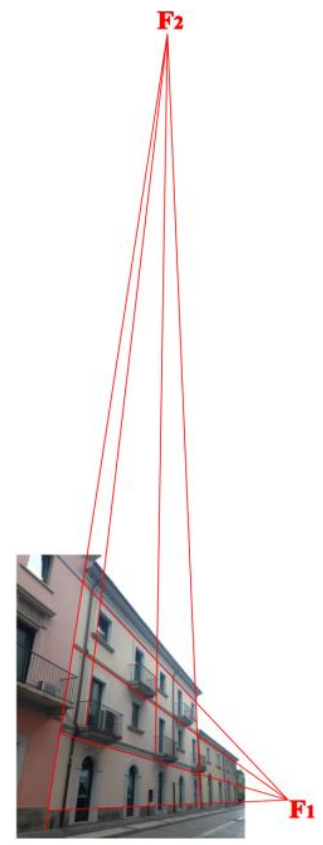

Figure 3. The classic method of Gaspard Monge.

Having an own software realized without the use of third-party libraries, as in our case, has led to implement an algorithm highly customizable in which, instead of resorting to classical projective geometry, it appeals to analytic geometry with the use of matrices for the calculation of appropriate 
intersections of lines representing the lines of flight[3].

\subsection{Principles of operation}

Just for this last reason, the software identification of vanishing lines must follow a particular process: that is identified on the four lines of escape from the first to the left or indistinctly drawn from the top down or from the bottom the high: it can indicate the entire left side of the facade or a part of it if not totally visible.

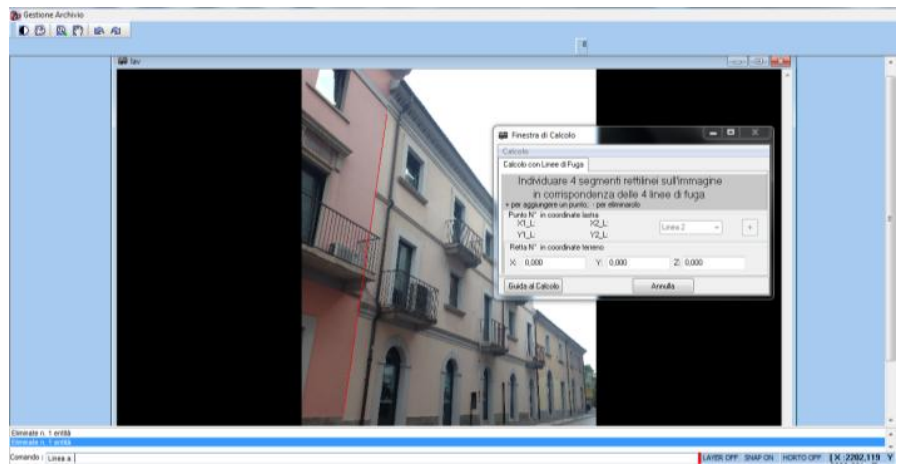

Figure 4. Identification of the first line of flight on the front on the left side of the image.

Then we proceed clockwise identifying from time to time the intersection between the two lines of flight streak. In this way, we avoid resort to improper points obtained by the line joining the vanishing lines in $\mathrm{x}$ and in $\mathrm{y}$.
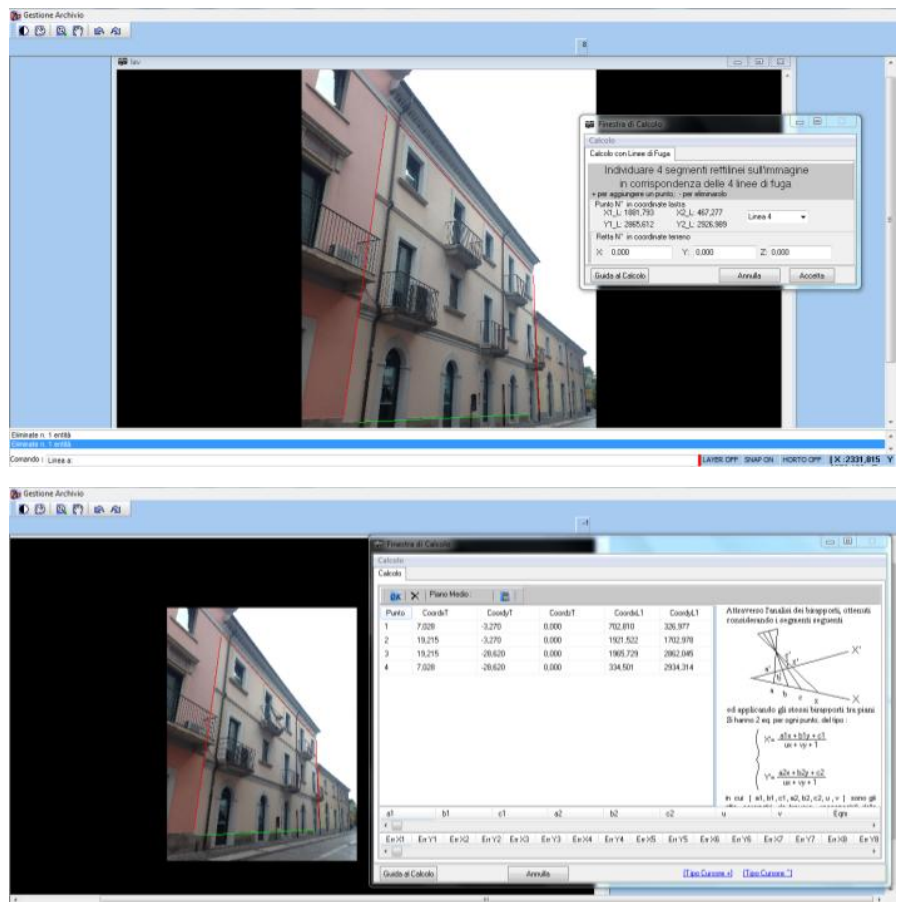

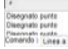

Figure 5-6. Calculation of the eight parameters of the homographic transformation.

In fact, you can see that by taking the projective rules, you would have the absurd that the more the photo is foreshortened longer valid values are obtained as the point determined by the improper joining the lines of flight with increasing inclination of these the past is better identified. Then work on a photo taken from the front would lead to absurd a major difficulty being the vanishing lines almost parallel assuming infinitely large values.

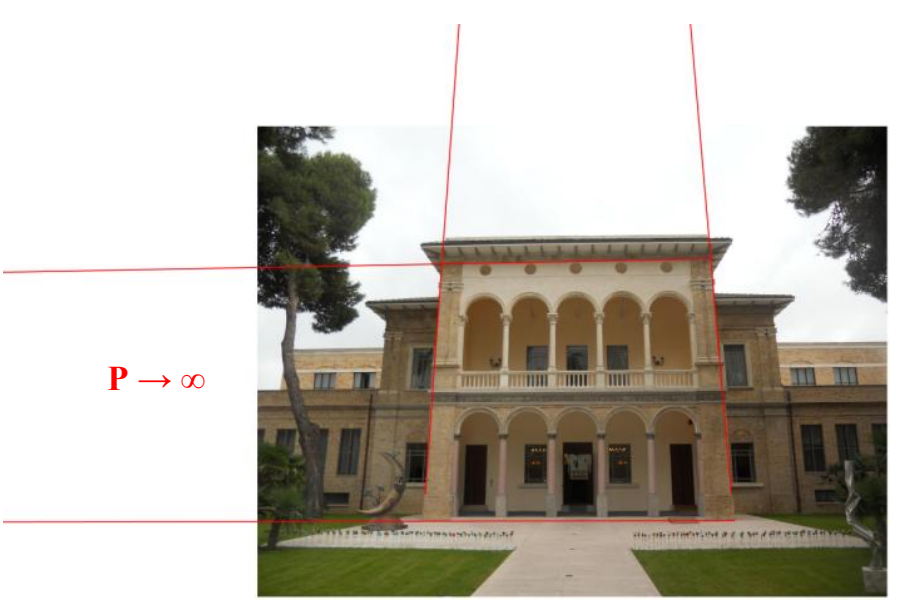

Figure 7. Parallel lines flight.

Following the procedure of the vanishing lines, it is clear that you lose the scale of the drawing as it was not introduced any comparison element having a known measurement both in length and in height.

It will be recalled that the image you are working in pixels; for this reason, before taking the picture of a prospectus it is good to put it on the references of note lengths.

Proposed in the application example, we know that the horizontal distance between the top of the lower external facade is $16.535 \mathrm{~m}$, while it is possible to note that the measure in the "return" is of another entity, which is equivalent to 12.215 units.

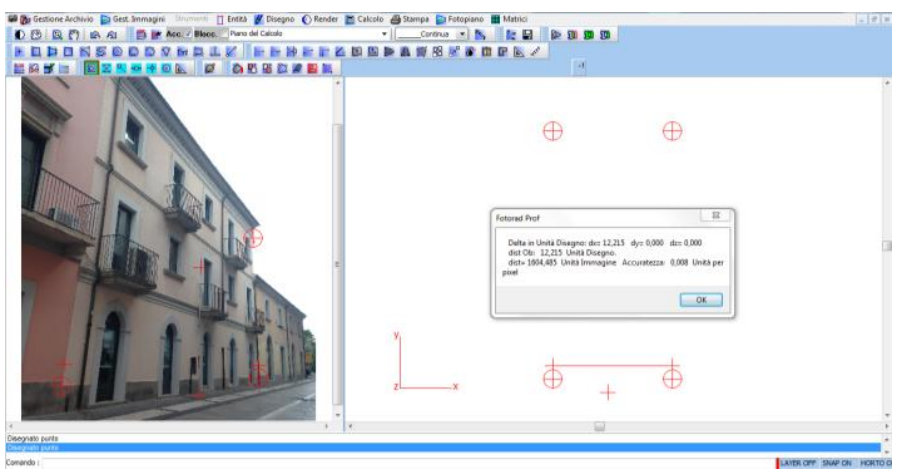

Figure 8. Identification of a horizontal measure known.

The same goes for the height, the distance between the top of the base and the apex in the upper left corner of the pilaster top is equal to 4.93 meters, while it is possible to note that the measure in the "return" is equal to 14.564 units.

It should at this point scale the drawing before starting the restoration of the facade.

It's possible to note how in scaling occurred, the known distances are scaled to be equal to those measured. 


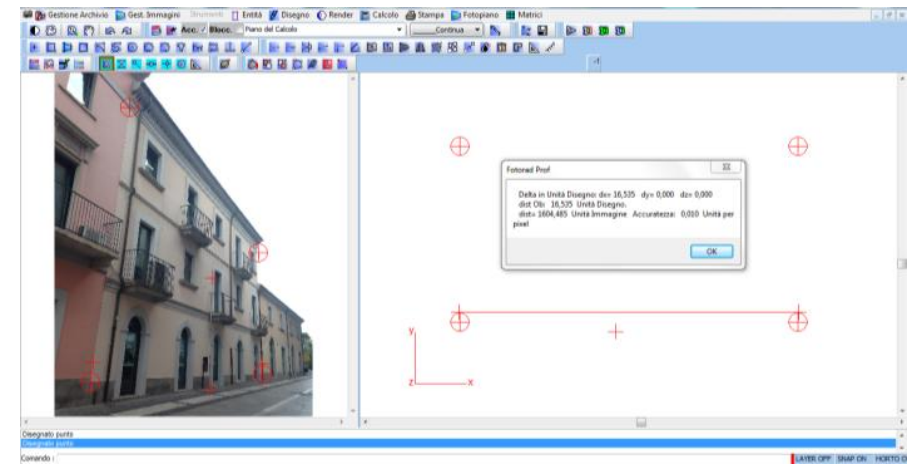

Figure 9. The horizontal measure of the window return is equal to the actual size of the facade.

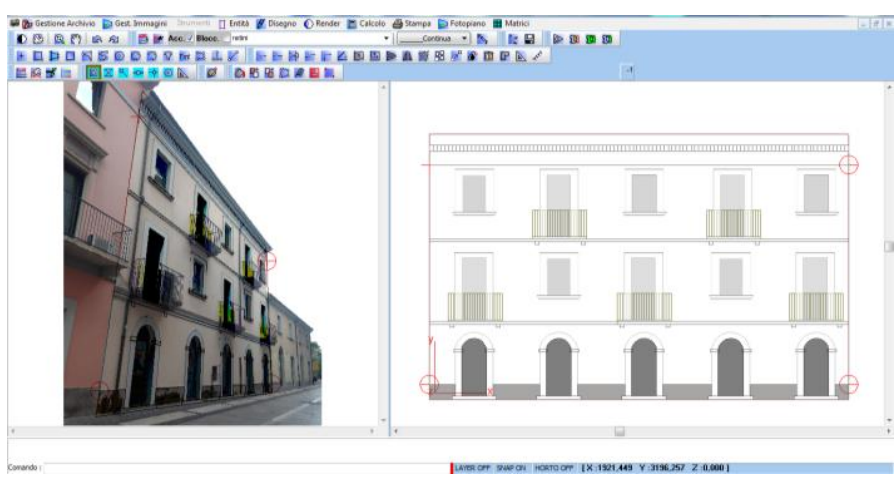

Figure 10. The final straightening made by the Engineering student Alessandro Biasco by the software Fotorad.

It 'clear that if the photo was taken from the front, the restitution of the façade presents no particular problems inherent in the projections or indentations if not only for the scaling. If we analyze now what happens in the case of a foreshortened image by calculating lines of flight, it is known as:

(1)Because of foreshortening is necessary to perform a stretching of pixels which is very large at the expense of the resolution of the image itself;

(2)Because it is lost the perception of perspective on the part of the designer who is no longer able to visually manage which parts lying on the ground and not preferential return.

\section{CONCLUSIONS}

As above, it is preferred to carry out the software Fotorad with two windows: the first contains the photo of foreshortening on which to draw; in the second, to return where you can view the drawing in vector format of the prospectus in orthogonal projection. In this way, with two windows, interactive between them, you can draw pictures on the fore foreshortened perspective in obtaining the orthogonal projection, the projection vice versa can be obtained as the inverse perspective transformation.

This allows you to have a further check on the accuracy of the return.

Faith without works is dead. Paraphrasing the biblical passage, we can say that talking about computer graphics means not only knowing the algorithms of the design but try to make them with any kind of language, be-cause only in this way you are able to grasp the subtleties of mere theory.

\section{REFERENCES}

[1] Bezoari, G. \& Monti, C. \& Sevini A. 1992. La fotogrammetria per l'architettura. Napoli: Liguori.

[2] Docci, M. \& Migliari, R. 1992. Scienza della rappresentazione. Fondamenti e applicazioni della geometria descrittiva. Roma: Carocci Editore.

[3] Palka, P. \& Cavallini, E. 2013. Informatica applicata al disegno automatico. Roma: Aracne Editrice.

[4] Rogers, D.F. \& Adams, J.A. 1990. Mathematical elements for computer graphics. Columbus: Mc-Graw Hill. 